\title{
Study on Sound Design in Films Based on Visual Image
}

\author{
Lu Dong \\ Harbin Institute of Technology, Harbin, 150001, China
}

Keywords: Visual image, Film, Sound design, Sound processing technology

\begin{abstract}
In films based on visual image, films are transformed into a medium combining auditory sense and visual sense from pure visual communication medium and a relative space-time structure shown by the combination of visual sense and auditory sense is transformed through silent films shown by visual sense. Sound becomes one of the important elements in film creation. However, from the perspective of subconsciousness of human beings, sound plays an auxiliary role in film works based on visual image mainly because people lack full understandings of functions of sound in films. This paper studies sound design in films based on visual image.
\end{abstract}

\section{Introduction}

In film sound design, sound engineer conducts technical processing of sound and the plot design of writer and director and the performance of actors are integrated. Though professional sound design work is not set in film sound design, sound design is very important for film play effect. Sound design is a complicated technical process. With sound element, its functions produce certain supporting capability for film story. Workers are required to exert professional technological level and meanwhile guarantee the coordination of sound and film contents. From the perspective of academic research, sound design is not defined very clearly and there are different opinions on its classification. Generally, sound design is put under the work of director. However, director pays more attention to the story and the overall effect of film design. Sound can be mastered in combination with film frame. It is very difficult to guide the application of sound element from professional perspective. In film creation, sound engineer designs sound according to the content. Therefore, sound design process is a procedural process and sound engineer masters each link in the application of sound element in Chinese film creation.

\section{Film sound design and sound processing technology}

\section{Film sound design}

The proposal of film sound design concept has necessary connection with the development of electronic science and technology. With the general application of electronic musical instrument, importance has been attached to sound processing technology and film sound design has emerged. In 1970s, sound design worker in sound production in American films was defined as Sound Designer. Work contents of sound design include the full application of sound art modeling technique and the completion of building of sound art image in film seeing and hearing work. Currently, there are film sound design studios at home and abroad. Sound designers working in such studios mostly use film post production software such as Pro tools and Nuendo for sound production and synthesis after technical processing of film sound element. Sound processing technology is adopted for language editing, environmental background sound and synthetic sound effect ${ }^{[1]}$.

\section{Sound processing technology}

Sound processing technology refers to technical processing of sound according to the need which causes the change of its physical properties. Physical properties of sound include length, pitch, tone and intensity of sound. In technical processing of sound, electronic synthesizer is used for electronic 
technical processing of sound and sound track is produced through sound sampling. Physical properties of sound change in the process of technical processing of sound.

In the rapid development process of electronic technology, sound processing technology has penetrated into application area widely and technical processing of film sound has promoted high-level development of film sound effect. Sound recording technology, effect processing technology, sound synthesis and modification technology are subject to parameter processing. Sound technologies are subdivided in this way, which is conducive to their effective application in application area.

\section{Selection of sound montage according to visual image}

The sound effect that can be achieved by sound montage can render visual image. The combination of sound and picture can make film contents more expressive. Sound montage refers to technical processing of sound such as cut and combination. Space-time is used as the unit in the processing process, thus making sound and film frame coordinating. Sound montage can be divided into narrative sound montage and expressive sound montage based on different sound processing technologies. Narrative sound montage is divided into sound advance, lag and conversion and synchronization of sound and picture in application skills and it is objective. Expressive sound montage focuses on the improvement of picture expression. It is divided into pure subjective sound, pure enjoyable sound, counterpoint of sound and picture, sound silence and emptiness and it is subjective. To use sound montage reasonably, it is required to get enlightenment from visual image.

Narrative sound montage is generally used for life pole of visual image. The method of sound advance can produce a tense atmosphere for films, accelerate scene change rhythm and make sound and scene consistent in representation style. Let's take Bourne Identity 3 for example. Landy did not impose a direct threat to Bern in the process of chasing Bern and it was life pole. At that time, Landy's sound delayed and covered Bern's scene. Therefore, a tense atmosphere was created under the action of sound.

Expressive sound montage is generally used for death pole of visual image so as to highlight the change of mood of characters. For example, when a killer chased Nicky and Nicky was escaping and felt that her life was threatened and she was faced with a crisis in Bourne Identity 3, sound montage technology was used to empty sound and audiences mostly heard twitter. The sound of music was deep. To render the sinister environmental atmosphere, subjective sound effect "whir" was added in the sound. Such sound made the picture effect more depressive and audiences seemed to feel that Nicky would leave here like a bird.

Sound montage should be selected according to film picture in the application process. Technical processing of sound based on visual image can produce a strong artistic effect of film.

\section{Matching between film sound and polarity of visual image}

The polarity of drama can be determined accurately according to script analysis. It is required to divide the polarity of film sound, establish a list of sounds in the two poles, make each polarity manifest the theme appropriately and guide reasonable sound collocation in film plot. Polarities generally existing in film plot include money and love, life and death, success and failure, rationality and irrationality etc. The existence of such polarities in a film can improve its visual effect. The contribution of sound design cannot be neglected.

The theme of a film often can be obtained through visual image. The relationship established among different themes can be analyzed. Contents of different themes in a film can be extended to language environment through sound effect. Music processing can make emotional tone to be expressed by the film more visual. In the theme to be expressed by the film, the key word of life pole is life and that of death pole is death. Both life and death are things revered by human beings and facts to be faced directly which cannot be changed. In a film, the performance of film characters requires the coordination of sound in front of the choice between life and death so as to make the theme to be 
expressed just right and allow audiences to have deeper understandings of film contents ${ }^{[2]}$. For spy films, the expression of life and death is the most critical plot. In terms of visual image design, it is required to determine key words of the plot and choose appropriate sound from the list of sounds in the two poles. In the real life, it is likely to choose a sound of "life pole" for images of death so as to manifest the expectation of the character for survival or the existence of hope for survival. However, a sound of "death pole" is often chosen for such technical processing of sound in most films and sounds of "life pole" that might exist due to environmental need are deleted so as to achieve good narrative effect. For example, when a character in the restaurant is in the scene of life-or-death choice, if the character does not have the threat of death, the background music is "life pole" at this time and audiences can hear objective music of the restaurant which gives people a sense of ease and allows them to relax naturally. From the perspective of film scene design, restaurant is a place of leisure, where people can enjoy themselves. When the film enters the plot of death pole, the relaxed objective music in the restaurant and the plot are inharmonious. At this time, the background music of life pole in the restaurant should be deleted and replaced by the film music with tense rhythm so as to present a sense of tension and solemnity. The overall style is more coordinating.

To strengthen the narrative effect of film, sound design should meet the polarity of visual image. For example, when a character is pushed to the edge of death, sounds representing death should occur. In Bourne Identity 3, when the killer was chasing Nicky, the background sound changed as the killer pushed the door. It seemed as space transition. The original background sound became deep and slow and the whole picture gradually became quiet. Only the cry of a child could be heard. This is a sound matching with death pole. As the cry of the child was highlighted, it seemed that the space of the scene was expanded and indicated that the character was going to death.

When a character is unexpectedly rescued from the edge of death, life pole element can occur and heartbeat indicates that the character faced with death has a favorable turn to life. When the character is faced with crisis and seems to have no opportunity for life, the emergence of heartbeat background music indicates that he has hope for life and his life does not end yet. Visual image and the two poles are not necessarily corresponding in special effect processing of film. They should be handled skillfully according to the change of film plot, thus giving audiences a sense of suspense and making film plot complicated ${ }^{[3]}$.

\section{Master overall trend of sound through visual image}

In films, visual image makes the thread of a story more visual. The effective combination of visual image and film theme through music design can determine the fundamental key of the film and allow audiences to judge the theme of the film and the development of story through the analysis on background sound. When the development process of story enters the stage of death pole of visual image, expressive sound montage can be used to mitigate and empty sound subjectively or silence can be adopted. Correspondingly, language rhythm should be accelerated and tone should be higher so as to highlight the tense atmosphere expressed in the plot. When it enters the stage of life pole of visual image, objective narrative sound montage should be used and everything in the film is normal, including pitch and language rhythm. To reflect the plot appropriately, more attention is paid to the quality of sound effect. At this time, sound rhythm, speed, advance and lag should coordinate with the scene skillfully so as to reflect life pole more smoothly.

Visual image plays a guiding role in film music. Subjective music can enrich and foil the theme of the film. Objective music is used for foiling environmental atmosphere, mainly including scene music. Subjective music manifests life pole of visual image. Soothing music is generally used, but the music sound is deep and gives people a sense of stateliness. Subjective music reflecting death pole is more shocking and gives people a cramped feeling and sense of depression about environmental atmosphere through tense rhythm. For example, when the killer occurred, a tense atmosphere was created with tense and shocking music. Objective music can foil the scene of film. For example, music in the restaurant and the environment of a coffee house can make audiences relaxed psychologically. With soothing music, audiences won't have a feeling of tension. In such a scene, a 
dignified atmosphere can be created when characters appeared seriously ${ }^{[4]}$. In addition, the quality of background sound has extremely strong expressive force for visual image. When characters are in a dangerous situation, music should be weakened or background music can be deleted. Subjective music can be used, such as the sound of fight and crush which can make the scene so quiet that people might feel nervous. Sounds with striking contrast can be used. For example, the sound of air and breath can be heard in the quiet environment. In particular, strengthening the sound of breath when a character is being chased is more helpful for creating a tense atmosphere.

Visual image and film sound can complement each other and meanwhile have repulsive interaction. This requires making a choice between them based on film contents so as to highlight scene effect. For example, music should be deleted in a scene of fight. Music should be used in the process of mutual chase so as to avoid dull scene. However, visual effect is mainly highlighted in the link of fight. Therefore, to avoid the influence of music, background music should be weakened or deleted so as to manifest the scene of severe fight fully.

\section{Conclusion}

In conclusion, essential properties of film have changed and film has gradually become an independent subject with the constant penetration of scientific and technological elements into film production. In film art, the transformation from visual art to visual and audio art makes film sound design a key link in the improvement of film quality. Sound processing technology consists in comprehensive application of sound, language and music so as to highlight the theme to be expressed by the film. Visual image plays a guiding role in film sound design. Visual image is required to provide skills and methods for both sound montage and sounds in the two poles so as to master the law of sound design better.

\section{References}

[1] [USA] Compiled by Ric Viers. Sound Effect Bible-Hollywood Sound Effect Creation and Recording Skills. Beijing: World Publishing Corporation, 2012: 134-148.

[2] Yao Rui, Yao Qiang. Digital Film Sound-Concept, Creativity and Development of All-dimension Surround Sound (ADSS) in 3D Era. Journal of Beijing Film Academy, 2012(05): 134-148.

[3] Wang Yueping. Analysis and Extraction of Flash Film Sound Features. Academic Discussion, 2015(07): 85-89.

[4] Wu Liying. Application of Sound Processing Technology in Film Sound Design. 2013 Audio Engineering Academic Forum and Annual Academic Communication Symposium, 2013:185-189. 